\title{
Адиабатический рэтчет-эффект в системах с дискретным изменением переменных
}

\author{
В. М. Розенбаум ${ }^{+1)}$, И. В. Шапочкина*, Л. И. Трахтенберг ${ }^{\times \circ}$ \\ + Институт химии поверхности им. А. А. Чуйко НАН Украины, 03164 Киев, Украина \\ * Белорусский государственный университет, физический факультет, 220050 Минск, Беларусь \\ × Федеральный исследовательский центр химической физики им. Н. Н. Семенова РАН, 119991 Москва, Россия
}

○ Московский государственный университет им. М. В. Ломоносова, 119991 Москва, Россия

\author{
Поступила в редакцию 15 мая 2020 г. \\ После переработки 8 августа 2020 г. \\ Принята к публикации 8 августа 2020 г.
}

\begin{abstract}
Развита адиабатическая теория рэтчет-эффекта в различных системах, описываемых случайными переходами между дискретными состояниями. В основе теории лежит построение дискретного аналога леммы Паррондо - одного из фундаментальных соотношений теории рэтчет-систем, позволяющего рассчитывать интегральные потоки за промежутки времени от начальных к конечным (равновесным) распределениям. Предложено соответствие между вероятностями переходов и параметрами потенциального рельефа, прыжковое движение в котором описывается развитой теорией и является низкотемпературным пределом непрерывного движения. Скорость рэтчет-эффекта, рассчитанная с помощью предложенной теории, хорошо подтверждается результатами численного моделирования. Развитый подход позволяет исследовать закономерности функционирования броуновских моторов различной природы простыми методами.
\end{abstract}

DOI: $10.31857 / \mathrm{S} 1234567820170103$

Под броуновским рэтчетом обычно понимают находящуюся в контакте с термостатом наносистему, в которой направленное движение возникает в результате несмещенных (unbiased) неравновесных возмущений различной природы при нарушении пространственной и/или временной симметрии [1-5]. Сам феномен возникновения направленного движения при таких условиях называют рэтчет-эффектом. Глубокое понимание механизма последнего возникает из рассмотрения его элементарного акта, описываемого известной леммой Паррондо [6]. В периодическом потенциальном профиле при заданной начальной плотности вероятности распределения частиц возникает поток частиц, который приводит с течением времени к установлению в системе термодинамического равновесия, характеризуемого больцмановской функцией распределения. Лемма Паррондо определяет интегральные потоки через заданные поперечные сечения за промежутки времени от начальных к конечным (равновесным) распределениям, что позволяет аналитически представлять решения сложных моделей, сводящиеся при тех или иных предположениях, к последовательности элементарных актов, описыва-

\footnotetext{
1) e-mail: vik-roz@mail.ru
}

емых леммой [7-10]. Один из механизмов функционирования рэтчета использует дихотомный процесс переключения асимметричных пространственно периодических потенциальных профилей $V_{\mathrm{A}}(x)$ и $V_{\mathrm{B}}(x)$ $\left[V_{\mathrm{A}(\mathrm{B})}(x+L)=V_{\mathrm{A}(\mathrm{B})}(x), L-\right.$ период]. Предполагая, что потенциалы переключаются мгновенно, а времена жизни профилей, $\tau_{\mathrm{A}}$ и $\tau_{\mathrm{B}}$, много больше характерных времен релаксации (адиабатическое приближение), среднюю скорость рэтчета можно определить через сумму двух интегральных потоков с начальными и конечными распределениями, соответствующими распределениям Больцмана $\rho_{-}^{\mathrm{A}(\mathrm{B})}(x)$ в профиле $V_{\mathrm{A}}(x)$ или $V_{\mathrm{B}}(x)$

$$
\begin{aligned}
& \langle\nu\rangle=\frac{L}{\tau_{\mathrm{A}}+\tau_{\mathrm{B}}} \int_{0}^{L} d x\left[\rho_{+}^{\mathrm{A}}(x)-\rho_{+}^{\mathrm{B}}(x)\right] \int_{0}^{x} d y\left[\rho_{-}^{\mathrm{A}}(y)-\rho_{-}^{\mathrm{B}}(y)\right], \\
& \rho_{ \pm}^{\mathrm{A}(\mathrm{B})}(x)=\exp \left[ \pm \beta V_{\mathrm{A}(\mathrm{B})}(x)\right] / \int_{0}^{L} d y \exp \left[ \pm \beta V_{\mathrm{A}(\mathrm{B})}(y)\right],
\end{aligned}
$$

где $\beta=\left(k_{B} T\right)^{-1}, k_{B}$ - постоянная Больцмана, $T$ абсолютная температура (подробный вывод выражения (1), в том числе и при учете неадиабатических и инерционных поправок, дан в [7-10]). 
При низких температурах $\left(k_{B} T\right.$ много меньше барьеров потенциальных рельефов) движение частиц приобретает прыжковый характер. Такой характер движения присущ молекулярным (белковым) моторам [11-13], в которых связывание на поверхности броуновской частицы заряженного лиганда (АТФ аденозинтрифосфата) приводит к сильному изменению потенциальной энергии этой частицы в асимметричном электростатическом поле полярной подложки. При определенных условиях, накладываемых теорией симметрии [14], прыжковый характер движения может быть инициирован и сильным перераспределением заряда наночастицы под действием внешнего электрического поля [15]. С более общей точки зрения, необходима сильная связь внешнего процесса, вызывающего флуктуации потенциального профиля и, как следствие, флуктуации констант скоростей преодоления потенциальных барьеров, с внутренним процессом возникновения направленного движения в рэтчет-сиситеме [16]. Электроконформационное сопряжение прикладываемого электрического поля и потока ионов через биологические мембраны служит ярким примером наличия такой связи внешнего и внутреннего процессов [17]. Для описания случайных переходов системы между дискретными состояниями может использоваться периодическая одномерная прыжковая модель диффузии частиц между узлами бесконечной цепочки с основной областью из $N$ узлов, развитая Дерридой [18]. Задавая вероятности переходов, можно рассчитывать характеристики поведения различных систем: от потоков частиц через биологические мембраны $[17,19,20]$ до изменения величины капитала в парадоксальных играх Паррондо [21-26]. При $N=2$ количественные связи между результатами для, казалось бы, различных моделей - флуктуирующего потенциала [27], электроконформационного сопряжения [17] и "каталитического колеса" [20] - устанавливаются особенно просто [16].

Анализируя непрерывные и дискретные проявления рэтчет-эффекта, следует отметить, что хотя дискретное движение можно рассматривать как предельный случай непрерывного движения, рэтчетэффект может носить и чисто дискретный характер, как, например, при кинетическом описании переходов между конечным набором состояний или в теории игр, когда эффект состоит в определенной смене стратегий игры, обеспечивающей средний выигрыш. Очевидно, что должен существовать дискретный аналог леммы Паррондо, описывающий элементарный акт, ответственный за рэтчет-эффект с дискретным изменением переменных, который, од- нако, до сих пор не был получен. В настоящей работе приводится простой вывод дискретного аналога леммы Паррондо, а также развитая с его помощью адиабатическая теория рэтчет-эффекта в системах с дискретным изменением переменных. Рассчитанные с использованием этой леммы величины средней скорости направленного движения хорошо подтверждаются результатами численного моделирования. Это позволяет эффективно использовать лемму для исследования закономерностей функционирования броуновских моторов простыми методами.

Рассмотрим бесконечную цепочку узлов, нумеруемых целочисленной переменной $n$. Пусть из каждого узла $n$ частица может перейти в соседний узел $n+1$ направо с вероятностью $p_{n}^{r}$, в соседний узел $n-1$ налево с вероятностью $p_{n}^{l}$ или остаться в узле $n$ с вероятностью $p_{n}^{u}=1-p_{n}^{r}-p_{n}^{l}$. Обозначим через $P_{n}(t)$ вероятность того, что частица находится в узле $n$ в момент времени $t$. Считая моменты времени целочисленными (происходящими через равные промежутки времени $\Delta t$ ), запишем уравнение, определяющее вероятность найти частицу в последующий момент времени $t+1$ в том же узле $n$ :

$$
P_{n}(t+1)=p_{n-1}^{r} P_{n-1}(t)+p_{n}^{u} P_{n}(t)+p_{n+1}^{l} P_{n+1}(t) .
$$

Если трактовать рэтчет-эффект в терминах теории парадоксальных игр Паррондо, то величина $P_{n}(t)$ приобретает смысл вероятности иметь капитал $n$ после $t$ бросков игральной кости, а уравнение (2) задает эволюцию $P_{n}(t)$, т.е. позволяет рассчитать результативность ведения игры.

Естественно ограничить число разнородных узлов $n$ в общей прыжковой модели. Проще всего это сделать введением элементарной ячейки из $N$ узлов, нумеруемых внутри нее целочисленной переменной $m=1,2, \ldots, N$, а все бесконечное множество узлов $n$ определять трансляциями элементарной ячейки, т.е. полагать $n=m+N k$, где $k=0, \pm 1, \ldots$ - целочисленное число трансляций. Тогда вероятности $p_{n}^{i}$ $(i=r, l, u)$ можно считать периодическими функциями с периодом $N\left(p_{n+N}^{i}=p_{n}^{i}\right)$ и определять набором $p_{m}^{i}$ с $m=1,2, \ldots, N$. Кроме того, введем редуцированную вероятность $R_{m}(t)=\sum_{k=-\infty}^{\infty} P_{m+N k}(t)$ заполнения состояния $m$ в момент времени $t$ с нормировкой на элементарную ячейку, $\sum_{m=1}^{N} R_{m}(t)=1$, которая является периодической функцией $R_{n}(t)=R_{n+N}(t)$ целочисленного аргумента $n$. В терминологии теории игр величина $R_{m}(t)$ задает вероятность иметь капитал $n$ по модулю $N$ (остаток от целочисленного деления $n$ на $N, m=n \bmod N)$. 
Замена индексов $n$ в уравнении (2) на $m+N k$ и суммирование по $k$ с использованием периодичности $p_{n+N}^{i}=p_{n}^{i}(i=r, l, u)$ приводит к уравнению на редуцированные вероятности $R_{m}(t)$, имеющему вид уравнения непрерывности:

$$
R_{m}(t+1)-R_{m}(t)=-\left[J_{m}(t)-J_{m-1}(t)\right]
$$

с дискретными потоками

$$
J_{m}(t)=p_{m}^{r} R_{m}(t)-p_{m+1}^{l} R_{m+1}(t) .
$$

Стационарное решение уравнения $(3) R_{m}(t)=$ $=R_{m}^{(\mathrm{st})}$ предполагает независимость потока от номера узла, $J_{m}^{\text {(st) }}=J_{m-1}^{\text {(st) }} \equiv J$, что дает разностное уравнение рекуррентного типа $p_{m}^{r} R_{m}^{(\mathrm{st})}-p_{m+1}^{l} R_{m+1}^{\text {(st) }}=$ $=J$, которое для новой неизвестной величины $\tilde{\xi}_{m} \equiv$ $\equiv p_{m}^{r} R_{m}^{(\mathrm{st})}$ и функции целочисленного аргумента $s_{m} \equiv p_{m}^{l} / p_{m}^{r}$ можно переписать как:

$$
\tilde{\xi}_{m}=s_{m+1} \tilde{\xi}_{m+1}+J .
$$

Проведя последовательные итерации и используя равенство $\tilde{\xi}_{m+N}=\tilde{\xi}_{m}$, уравнение (5) легко преобразовать к виду:

$$
\left(1-\prod_{n=1}^{N} s_{n}\right) \tilde{\xi}_{m}=J \xi_{m},
$$

где введено обозначение

$$
\xi_{m} \equiv 1+\sum_{n=1}^{N-1} \prod_{j=1}^{n} s_{m+j} .
$$

Дополним уравнение (6) с двумя неизвестными величинами $\tilde{\xi}_{m}$ и $J$ условием нормировки $\sum_{m=1}^{N} R_{m}^{(\mathrm{st})}=$ $=\sum_{m=1}^{N} \tilde{\xi}_{m} / p_{m}^{r}=1$. Тогда искомые величины определятся выражениями:

$$
\begin{gathered}
J=\left(\sum_{n=1}^{N} \frac{\xi_{n}}{p_{n}^{r}}\right)^{-1}\left(1-\prod_{n=1}^{N} s_{n}\right), \\
R_{m}^{\text {(st) }}=\left(\sum_{n=1}^{N} \frac{\xi_{n}}{p_{n}^{r}}\right)^{-1} \frac{\xi_{m}}{p_{m}^{r}}
\end{gathered}
$$

впервые представленными в [18]. Из первого соотношения (8) следует, что термодинамическое равновесие может реализоваться только при определенном условии, накладываемом на вероятности переходов:

$$
\prod_{n=1}^{N} s_{n}=1, \quad p_{1}^{r} p_{2}^{r} \ldots p_{N}^{r}=p_{1}^{l} p_{2}^{l} \ldots p_{N}^{l},
$$

известном как условие детального баланса.
Соотношения (8) являются дискретными аналогами известного результата Стратоновича, описывающего диффузию броуновской частицы в периодическом потенциале под действием стационарной однородной силы $[28,29]$. Чтобы убедиться в этом, используем подход работы [30] и представим, что каждому узлу $m$ соответствует потенциальная яма со значением минимума $u_{m}$, окруженная барьерами со значениями максимумов $v_{m}$ справа и $v_{m-1}$ слева. Введем такой наклон потенциального рельефа, что потенциальные барьеры слева и справа получают приращения $f$ и $-f$ соответственно (рис. 1a). Если измерять введенные энергетические величины в единицах $k_{B} T$, то вероятности преодоления барьеров можно отождествить с аррениусовскими факторами:

$$
p_{m}^{l}=e^{-v_{m-1}+u_{m}-f}, \quad p_{m}^{r}=e^{-v_{m}+u_{m}+f} .
$$

Подстановка (10) в (8) дает:

$$
\begin{aligned}
J & =\frac{1-e^{-2 N f}}{\sum_{n=1}^{N} e^{-u_{n}+(2 n-1) f} \sum_{k=n}^{n+N-1} e^{v_{k}-2 k f}}, \\
R_{m}^{(\text {st })} & =\frac{e^{-u_{m}+(2 m-1) f} \sum_{k=m}^{m+N-1} e^{v_{k}-2 k f}}{\sum_{n=1}^{N} e^{-u_{n}+(2 n-1) f} \sum_{k=n}^{n+N-1} e^{v_{k}-2 k f}} .
\end{aligned}
$$

Полученные дискретные соотношения соответствуют интегральным представлениям результата Стратоновича (см. формулы (2.36)-(2.38) обзора [1]), если учесть, что внутренним интегралам соответствуют внутренние суммы, содержащие значения максимумов барьеров (барьерные факторы), а внешним интегралам - внешние суммы, содержащие факторы, которые зависят от значений минимумов потенциальных ям. В отсутствие наклона потенциального рельефа $(f=0)$ поток исчезает $(J=0)$, барьерные факторы в стационарном распределении $R_{m}^{(\text {st) }}$ сокращаются, и оно переходит в равновесное распределение Больцмана $R_{m}^{(\mathrm{eq})}=e^{-u_{m}} / \sum_{n=1}^{N} e^{-u_{n}}$, как и должно быть.

Соответствие между параметрами потенциального рельефа и вероятностями переходов между узлами цепочки, задаваемое соотношениями (10), обладает глубоким физическим смыслом, поскольку позволяет рассматривать прыжковое движение как низкотемпературный предел непрерывного $[4,16,31,32]$. Этим оно отличается от формальных попыток определять дискретные точки рельефа по соответствию 
(a)
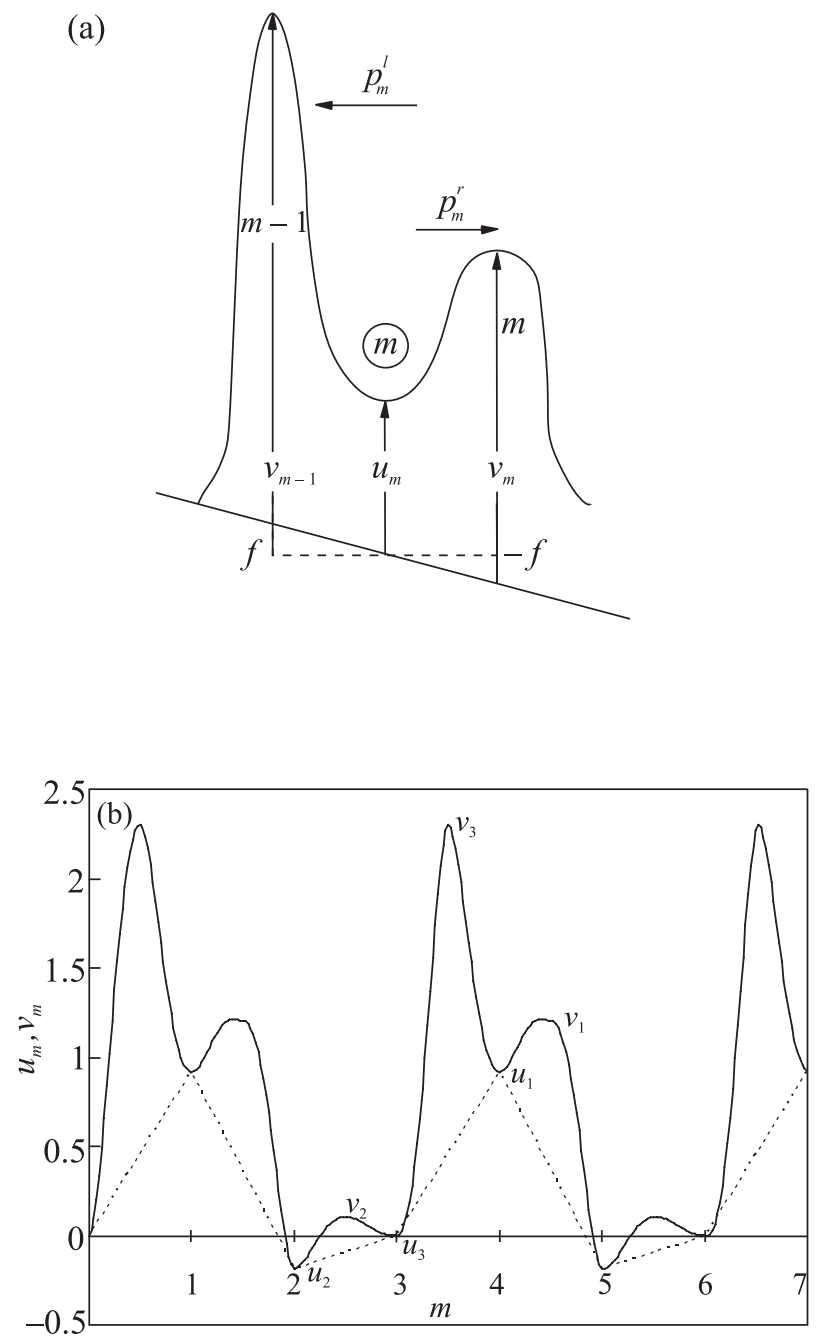

Рис. 1. (а) - Форма потенциального рельефа вблизи узла $m$, обеспечивающая переходы частицы в соседние узлы с вероятностями $p_{m}^{r}$ и $p_{m}^{l}$, зависящими от параметров рельефа по закону Аррениуса. (b) - Потенциальный рельеф с экстремумами $u_{1}=\ln (5 / 2)$, $u_{2}=-\ln (6 / 5), u_{3}=0, v_{1}=\ln (10), v_{2}=\ln (10 / 3)$, $v_{3}=\ln (10 / 9)$, рассчитанными по формулам (10) с параметрами $p_{1}^{r}=p_{2}^{r}=3 / 4, p_{1}^{l}=p_{2}^{l}=1 / 4, p_{3}^{r}=1 / 10$, $p_{3}^{l}=9 / 10$ парадоксальной игры Паррондо (сплошные кривые), и кусочно-линейный рельеф, представленный в [24] (пунктирные линии)

с коэффициентами уравнения Фоккера-Планка [24]. Следует иметь в виду, что система с $N$ узлами в элементарной ячейке характеризуется набором из $2 N$ параметров, которые могут задаваться вероятностями перескоков налево $p_{m}^{l}$ и направо $p_{m}^{r}$ или значениями минимумов $u_{m}$ и максимумов $v_{m}(m=1,2, \ldots, N)$ потенциального рельефа. На рисунке $1 \mathrm{~b}$ изображен потенциальный профиль с положениями экстремумов, рассчитанными по формулам (10) со значения- ми вероятностей, выбранных в парадоксальной игре Паррондо [21]. Подход работы [24], в котором наложено $N$ дополнительных условий $p_{m}^{u}=0$, не предполагает отображения барьерных участков, поэтому потенциальный профиль представляет собой ломаную линию, соединяющую минимумы потенциальных ям.

Перейдем к выводу дискретного аналога леммы Паррондо. Введем обозначения $\Phi_{m}(\tau)$ и $\varphi_{m}(\tau)$ для искомого интегрального потока и вспомогательной интегральной вероятности соответственно:

$$
\begin{aligned}
\Phi_{m}(\tau) \equiv \sum_{t=0}^{\tau-1} J_{m}(t) & =p_{m}^{r} \varphi_{m}(\tau)-p_{m+1}^{l} \varphi_{m+1}(\tau), \\
\varphi_{m}(\tau) & =\sum_{t=0}^{\tau-1} R_{m}(t) .
\end{aligned}
$$

Лемма Паррондо позволяет выразить $\Phi_{m}(\tau)$ через $R_{m}(0)$ и $R_{m}(\tau)$, минуя непосредственное вычисление $\varphi_{m}(\tau)$. При произвольных $\tau$ использование леммы дает незначительное упрощение вычислений, поскольку нахождение редуцированной вероятности $R_{m}(\tau)$ все равно потребуется. Однако при больших $\tau$ упрощение вычислений существенно, вплоть до возможности аналитического получения решений для ряда ситуаций, поскольку $R_{m}(\tau)$ стремится к равновесному распределению Больцмана $R_{m}^{(\mathrm{eq})}$, вид которого известен. Покажем, как возникает такая оптимизация преобразований.

Суммирование уравнения непрерывности (4) по дискретной временной переменной $t$ от 0 до $\tau-1$ дает уравнение $R_{m}(\tau)-R_{m}(0)=-\left[\Phi_{m}(\tau)-\Phi_{m-1}(\tau)\right]$, которое при последующем суммировании по $m$ от 2 до $n$ приводит к формуле

$$
\Phi_{n}(\tau)=\Phi_{1}(\tau)+\sum_{m=2}^{n}\left[R_{m}(0)-R_{m}(\tau)\right],
$$

позволяющей выразить интегральный поток в произвольном узле $n>1$ через интегральный поток в узле $n=1$. Для вычисления $\Phi_{n}(\tau)$ используем явное выражение, задаваемое формулой (12), представив его в удобном для дальнейших преобразований виде:

$$
\Phi_{n}(\tau)=\alpha_{n}\left[\beta_{n} \varphi_{n}(\tau)-\beta_{n+1} \varphi_{n+1}(\tau)\right],
$$

где $\alpha_{n}=\alpha_{n+N}$ и $\beta_{n}=\beta_{n+N}-$ функции целочисленного аргумента, подлежащие определению. Фактор в квадратных скобках является аналогом полного дифференциала от периодической функции и обращается в нуль при суммировании по элементарной ячейке из $N$ узлов в силу условия периодичности $\beta_{1} \varphi_{1}(\tau)=\beta_{N+1} \varphi_{N+1}(\tau)$. Функции $\alpha_{n}$ и $\beta_{n}$ удовлетворяют системе уравнений $\alpha_{n} \beta_{n}=p_{n}^{r}, \alpha_{n} \beta_{n+1}=p_{n+1}^{l}$, 
которая сводится к уравнению $\alpha_{n}=s_{n+1} \alpha_{n+1}$, совпадающему с уравнением (5) для $\tilde{\xi}_{m}$ при $J=0$. Последнее означает, что в качестве решения $\alpha_{n}$ с точностью до произвольной постоянной $C$ можно использовать функцию $\xi_{n}$, задаваемую соотношением $(7) \mathrm{c}$ дополнительным условием $(9), \alpha_{n}=C \xi_{n}$. Действительно, непосредственной подстановкой этого решения в выражение $\alpha_{n}-s_{n+1} \alpha_{n+1}$ можно убедиться, что оно равно $C\left(1-s_{1} s_{2} \ldots s_{N}\right)$, т.е. $\alpha_{n}=s_{n+1} \alpha_{n+1}$ при $s_{1} s_{2} \ldots s_{N}=1$.

Подстановка выражения для интегрального потока (14) в (13), домножение результата почленно на $\alpha_{n}^{-1}$ и последующее суммирование по всем узлам элементарной ячейки обращает в ноль левую часть полученного уравнения, которая содержит интегральные вероятности $\varphi_{n}(\tau)$ и $\varphi_{n+1}(\tau)$. Тогда в правой части получившегося уравнения коэффициент пропорциональности $C$ между величинами $\alpha_{n}$ и $\xi_{n}$ сокращается, что и приводит к окончательному выражению для $\Phi_{1}(\tau)$ :

$$
\begin{gathered}
\Phi_{1}(\tau)=\sum_{n=2}^{N} Q_{n} \sum_{m=2}^{n}\left[R_{m}(\tau)-R_{m}(0)\right], \\
Q_{n}=\xi_{n}^{-1} / \sum_{m=1}^{N} \xi_{m}^{-1} .
\end{gathered}
$$

Использование выражений (15) в составе формулы (13) определяет $\Phi_{n}(\tau)$ при произвольном $n>1$. Сопоставление выражения для $Q_{n}$ в $(15)$ с параметрами эквивалентного потенциального рельефа, задаваемыми соотношением (10), дает $Q_{n}=e^{v_{n}} / \sum_{m=1}^{N} e^{v_{m}}$, откуда следует, что величины $Q_{n}$ определяются барьерными факторами, т.е. выступают эквивалентами функций $\rho_{+}^{\mathrm{A}(\mathrm{B})}(x)$ в непрерывном описании леммы Паррондо (1).

Соотношения (13), (15) являются точными и представляют основной результат данной статьи. Они позволяют рассчитывать интегральные потоки за произвольный промежуток дискретного времени $\tau$, если известна редуцированная вероятность $R_{m}(\tau)$. При больших временах $\tau$, когда устанавливается термодинамическое равновесие, $R_{m}(\tau)$ стремится к известному больцмановскому распределению $R_{m}^{(\mathrm{eq})}$, и соотношения (13), (15) становятся дискретным эквивалентом леммы Паррондо. Таким образом, на временной шкале результат (13), (15) имеет даже большую общность, нежели собственно лемма Паррондо. Значение полученного результата состоит в возможности изучать динамику средних значений заполнений узлов $n$ и характеристики рэтчет-эффекта при наличии флуктуаций вероятностей переходов меж- ду узлами (флуктуаций потенциального профиля в рэтчет-системах). При соблюдении условий детального баланса (9) и в отсутствие флуктуаций приращение средних значений $n$ определяется суммой интегральных потоков:

$$
\begin{gathered}
\langle n(\tau)\rangle=\sum_{n=-\infty}^{\infty} n P_{n}(\tau)=\langle n(0)\rangle+\sum_{m=1}^{N}\left(p_{m}^{r}-p_{m}^{l}\right) \varphi_{m}(\tau)= \\
=\langle n(0)\rangle+\sum_{m=1}^{N} \Phi_{m}(\tau) .
\end{gathered}
$$

При достаточно больших $\tau$, когда устанавливается термодинамическое равновесие, величины $\Phi_{m}(\tau)$ перестают зависеть от $\tau$ и возникает постоянное приращение $\langle n(\infty)\rangle-\langle n(0)\rangle$.

Рассмотрим процесс чередования двух состояний А и В с длительностями $\tau_{\mathrm{A}}$ и $\tau_{\mathrm{B}}$, характеризуемыми, соответственно, наборами вероятностей $p_{n, \mathrm{~A}}^{r}$, $p_{n, \mathrm{~A}}^{l}$ и $p_{n, \mathrm{~B}}^{r}, p_{n, \mathrm{~B}}^{l}$. Поскольку конечный момент времени одного состояния является начальным для другого $\left(\left\langle n_{\mathrm{A}}(0)\right\rangle=\left\langle n_{\mathrm{B}}\left(\tau_{\mathrm{B}}\right)\right\rangle\right)$, то, применяя (16) последовательно для $\left\langle n_{\mathrm{A}}\left(\tau_{\mathrm{A}}\right)\right\rangle$ и для $\left\langle n_{\mathrm{B}}\left(\tau_{\mathrm{B}}\right)\right\rangle$ и учитывая далее (13), получаем среднюю скорость изменения средних значений заполнений узлов $n$ за один временной цикл $\tau_{\mathrm{A}}+\tau_{\mathrm{B}}$ :

$$
\begin{gathered}
\langle v\rangle=\frac{\left\langle n_{\mathrm{A}}\left(\tau_{\mathrm{A}}\right)\right\rangle-\left\langle n_{\mathrm{B}}(0)\right\rangle}{\tau_{\mathrm{A}}+\tau_{\mathrm{B}}}= \\
=\left(\tau_{\mathrm{A}}+\tau_{\mathrm{B}}\right)^{-1} \sum_{m=1}^{N}\left[\Phi_{m, \mathrm{~A}}\left(\tau_{\mathrm{A}}\right)+\Phi_{m, \mathrm{~B}}\left(\tau_{\mathrm{B}}\right)\right]= \\
=\frac{N}{\tau_{\mathrm{A}}+\tau_{\mathrm{B}}}\left[\Phi_{1, \mathrm{~A}}\left(\tau_{\mathrm{A}}\right)+\Phi_{1, \mathrm{~B}}\left(\tau_{\mathrm{B}}\right)\right]
\end{gathered}
$$

или, после подстановки выражения (15), эта скорость есть

$$
\begin{gathered}
\langle v\rangle=\frac{N}{\tau_{\mathrm{A}}+\tau_{\mathrm{B}}} \sum_{n=2}^{N}\left(Q_{n, \mathrm{~A}}-Q_{n, \mathrm{~B}}\right) \times \\
\times \sum_{m=2}^{n}\left[R_{m, \mathrm{~A}}\left(\tau_{\mathrm{A}}\right)-R_{m, \mathrm{~B}}\left(\tau_{\mathrm{B}}\right)\right], \\
Q_{n, \mathrm{~A}(\mathrm{~B})}=\frac{\xi_{n, \mathrm{~A}(\mathrm{~B})}^{-1}}{\sum_{m=1}^{N} \xi_{m, \mathrm{~A}(\mathrm{~B})}^{-1}} .
\end{gathered}
$$

Здесь величина $\xi_{n}$ для состояний $\mathrm{A}(\mathrm{B})$ определена в (7). Отметим, что структура выражения (18) подобна (1), как и должно быть, поскольку (18) есть дискретный аналог выражения для скорости рэтчета в непрерывной модели.

Простейший пример, допускающий аналитическое представление редуцированной вероятности 
$R_{m}(t)$ с произвольным $t$, соответствует случаю $N=2$, при котором

$$
\begin{gathered}
R_{1}(t)=1-R_{2}(t)= \\
=\bar{R}_{1}^{(\mathrm{eq})}+\left[R_{1}(0)-\bar{R}_{1}^{(\mathrm{eq})}\right]\left(1-p_{1}-p_{2}\right)^{t}, \\
\bar{R}_{1}^{(\mathrm{eq})}=\frac{p_{2}}{p_{1}+p_{2}}, \quad p_{m}=p_{m}^{r}+p_{m}^{l}, \quad m=1,2 .
\end{gathered}
$$

Если вероятности оставаться в том же узле равны нулю, $p_{m}^{u}=0, p_{m}=1(m=1,2)$, то четность номера узла пребывания частицы будет изменяться после каждого прыжка, приводя к осцилляциям $R_{1}(t)=\bar{R}_{1}^{\text {(eq) }}+\left[R_{1}(0)-\bar{R}_{1}^{(\mathrm{eq})}\right](-1)^{t}$, не убывающим с ростом $t$. Поскольку $\Phi_{1}(\tau)=\left(p_{1}^{r} / p_{1}\right)\left[R_{1}(0)-R_{1}(\tau)\right]$ и

$$
\begin{gathered}
\sum_{m=1}^{2} \Phi_{m}(\tau)=\left[\left(p_{1}^{r}-p_{1}^{l}\right) / p_{1}\right]\left[R_{1}(0)-R_{1}(\tau)\right], \text { то } \\
\langle n(\tau)\rangle=\langle n(0)\rangle+ \\
+\left[\left(p_{1}^{r}-p_{1}^{l}\right) / p_{1}\right]\left[R_{1}(0)-\bar{R}_{1}^{(\mathrm{eq})}\right]\left[1-\left(1-p_{1}-p_{2}\right)^{\tau}\right] .
\end{gathered}
$$

Тогда при чередовании двух состояний А и В скорость рэтчет-эффекта равна:

$$
\begin{aligned}
& \langle v\rangle=\frac{2\left[\left(p_{1, \mathrm{~A}}^{r} / p_{1, \mathrm{~A}}\right)-\left(p_{1, \mathrm{~B}}^{r} / p_{1, \mathrm{~B}}\right)\right]}{\tau_{\mathrm{A}}+\tau_{\mathrm{B}}}\left[\bar{R}_{1, \mathrm{~B}}^{(\mathrm{eq})}-\bar{R}_{1, \mathrm{~A}}^{(\mathrm{eq})}\right] \times \\
& \times\left[1-\left(1-p_{1, \mathrm{~A}}-p_{2, \mathrm{~A}}\right)^{\tau_{\mathrm{A}}}\right]\left[1-\left(1-p_{1, \mathrm{~B}}-p_{2, \mathrm{~B}}\right)^{\tau_{\mathrm{B}}}\right] .
\end{aligned}
$$

На рисунке 2 теоретические зависимости (20), (21) сопоставлены с результатами моделирования, при котором случайные смещения частицы на каждом шаге дискретного изменения времени отрабатывались с помощью генератора случайных чисел в соответствии с исходным управляющим уравнением (2), а полученные траектории движения затем усреднялись. В качестве А выбрано симметричное состояние (аналог процесса свободной диффузии в отсутствие потенциального профиля), в котором $p_{\mathrm{A}}^{r}=p_{\mathrm{A}}^{l}=1 / 2$ вне зависимости от номера узла. Состояние В задано набором параметров $p_{1, \mathrm{~B}}^{r}=0.2, p_{1, \mathrm{~B}}^{l}=0.3, p_{2, \mathrm{~B}}^{r}=0.54, p_{2, \mathrm{~B}}^{l}=0.36$, a состояние $\mathrm{C}$ определено как антисимметричное к B, что соответствует параметрам с инвертированными индексами 1 и 2. Начальное условие выбрано как $\langle n(0)\rangle=R_{1}(0)=0$. Симметричное состояние $\mathrm{A}$ не дает смещения среднего значения заполнения узла $n$, т.е. $\left\langle n_{\mathrm{A}}(t)\right\rangle=\left\langle n_{\mathrm{A}}(0)\right\rangle=0$, согласно (20) при $p_{\mathrm{A}}^{r}=p_{\mathrm{A}}^{l}=1 / 2$. Смещения возможны для несимметричных состояний В и С. Формула (20) очень точно воспроизводит модельную зависимость $\left\langle n_{\mathrm{B}(\mathrm{C})}(t)\right\rangle$ (сравни положения маркеров относительно пунктирных линий на рис. 2a). Установившиеся
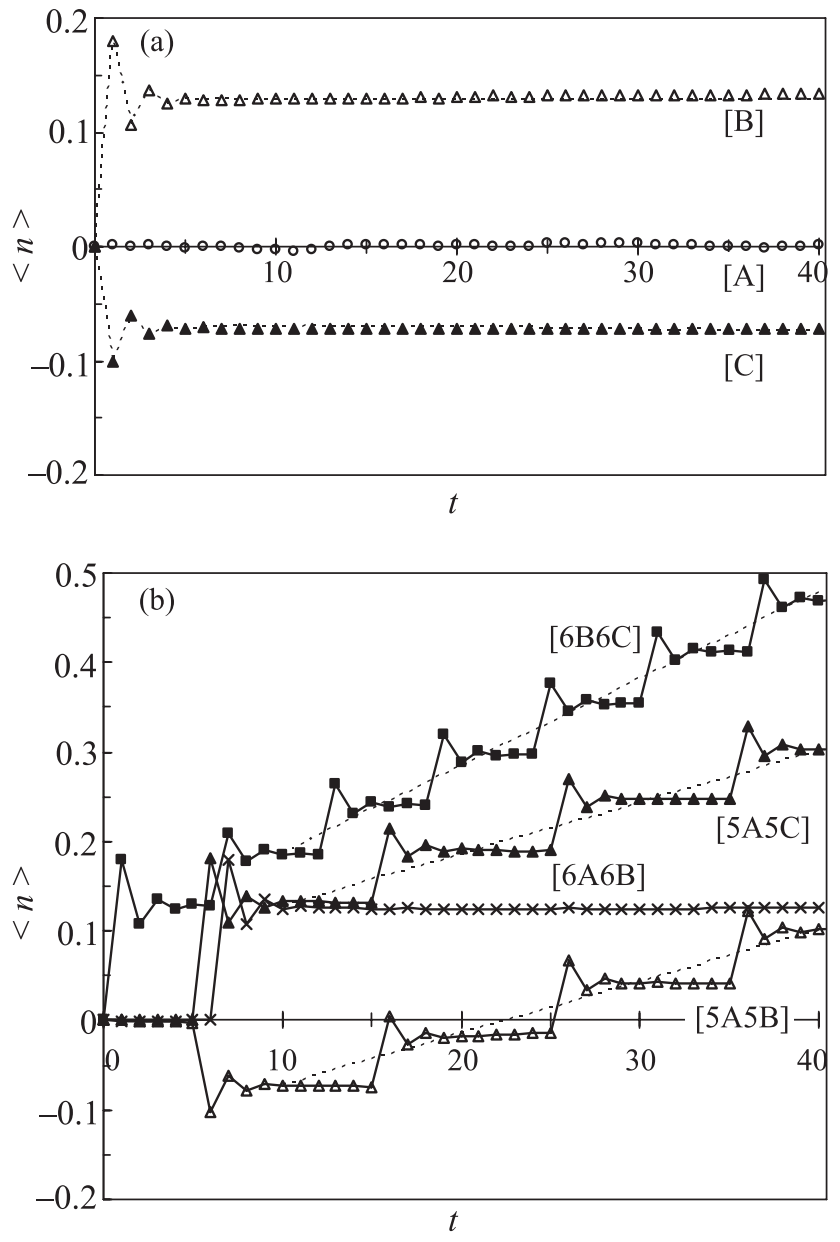

Рис. 2. Зависимости средних значений заполнений узлов $n$ от дискретного времени $t$, рассчитанные при $N=2$ путем численного моделирования с усреднением по 3 млн. траекторий (маркеры) и по формулам (20), (21) (пунктирные линии). Результаты для состояний A, В и С (описаны в тексте) представлены на рисунке (a), а при чередовании этих состояний - на рисунке (b) (символы в [...] описывают последовательность чередования, например, [6B6C] соответствует чередованию состояний В и С с длительностями $\tau_{\mathrm{B}}=6$ и $\tau_{\mathrm{C}}=6$ )

значения $\left\langle n_{\mathrm{B}}(\infty)\right\rangle=9 / 70$ и $\left\langle n_{\mathrm{C}}(\infty)\right\rangle=-5 / 70$ реализуются достаточно быстро: при $\tau>5$. Рэтчетэффект возникнет при чередовании состояний; исключение - реализация состояния А с четным значением его длительности $\tau_{\mathrm{A}}$ (рис. $2 \mathrm{~b}$ ). Значения скоростей рэтчет-эффекта, рассчитываемые по формуле (21) (пунктирные линии на рис. 2b), правильно описывают наклон модельных зависимостей.

Соотношения (13), (15), (16), (18) можно применить и к парадоксальной игре Паррондо (обозначаемой здесь как D с параметрами, представленными в подписи к рис. 1), соответствующей $N=3$ [21-25]. 
Средний капитал при достаточно больших $\tau>20$ выходит на значение $\left\langle n_{\mathrm{D}}(\infty)\right\rangle=-88 / 169$, также хорошо согласующееся с результатом моделирования (рис.3). Чередование игры D с симметричной иг-

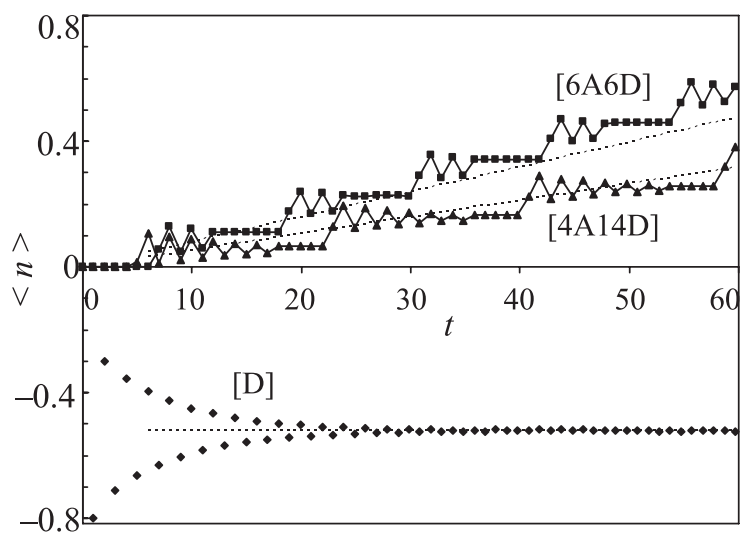

Рис. 3. Зависимости среднего капитала $\langle n\rangle$ от числа бросков игральной кости $t$ в случае $N=3$ для игры $\mathrm{D}$ и ее чередования с игрой А (парадоксальная игра Паррондо), при различных длительностях игр $\tau_{\mathrm{A}}$ и $\tau_{\mathrm{D}}$. Результаты моделирования с усреднением по 3 млн. траекторий и расчетами по формулам (16) и (18) представлены маркерами и пунктирными линиями

рой А приводит к рэтчет-эффекту, характеризующемуся скоростью изменения среднего капитала $\langle v\rangle=$ $=(16 / 169) /\left(\tau_{\mathrm{A}}+\tau_{\mathrm{D}}\right)$, которую демонстрирует и моделирование при достаточно больших $\tau_{\mathrm{D}}$ (сравни пунктирные линии и маркеры на рис. 3).

Таким образом, представленный в данной статье дискретный аналог леммы Паррондо, одного из фундаментальных соотношений теории рэтчетов, позволил развить теоретический аппарат рэтчет-эффекта в системах с дискретным изменением переменных, а также установить количественные связи между различными моделями. Полученное представление для дискретных систем является даже более общим, чем интегральная лемма Паррондо, поскольку применимо не только к адиабатическим системам. Основными параметрами предложенного описания являются вероятности переходов между узлами одномерной решетки, которые ставятся в соответствие величинам экстремумов потенциального рельефа, прыжковое движение в котором описывается предлагаемой теорией и является низкотемпературным пределом непрерывного движения. Соотношения для скорости дискретного рэтчет-эффекта в случаях двух и трех неэквивалентных узлов элементарной ячейки одномерной решетки приводят к результатам, находящимся в хорошем согласии с результатами численного моделирования. Представленные количественные связи параметров дискретных и непрерывных моде- лей позволяют учитывать влияние внешних процессов (например, конформационных переходов и перераспределений заряда под действием гидролиза АТФ или внешних электромагнитных полей) в терминах флуктуаций потенциального рельефа, вдоль которого движется броуновская частица, а значит исследовать закономерности функционирования броуновских моторов относительно простыми модельными методами.

Работа выполнена в рамках Государственного задания 0082-2018-0003 (регистрационный номер АAАА-А18-118012390045-2) и поддержана Российским фондом фундаментальных исследований (проекты 20-57-00007 и 18-29-02012-мк), Белорусским республиканским фондом фундаментальных исследований (проект Ф20Р-032).

1. P. Reimann, Phys. Rep. 361, 57 (2002).

2. P. Hänggi and F. Marchesoni, Rev. Mod. Phys. 81, 387 (2009).

3. D. Cubero and F. Renzoni, Brownian Ratchets: From Statistical Physics to Bio and Nanomotors, Cambridge University Press, Cambridge, UK (2016).

4. В. М. Розенбаум, И.В. Шапочкина, Л.И. Трахтенберг, Успехи физических наук 189, 529 (2019) [V. M. Rozenbaum, I. V. Shapochkina, and L. I. Trakhtenberg, Physics-Uspekhi 62, 496 (2019)].

5. Ю.В. Гуляев, А. С. Бугаев, В. М. Розенбаум, Л.И. Трахтенберг, Успехи физических наук 190, 337 (2020) [Yu. V. Gulyaev, A. S. Bugaev, V.M. Rozenbaum, and L. I. Trakhtenberg, PhysicsUspekhi 63(4), 311 (2020)].

6. J. M. R. Parrondo, Phys. Rev. E 57, 7297 (1998).

7. В. М. Розенбаум, И. В. Шапочкина, Письма в ЖЭТФ 92, 124 (2010) [JETP Lett. 92, 120 (2010)].

8. В. М. Розенбаум, И. В. Шапочкина, Т. Е. Корочкова, Письма в ЖЭТФ 98, 637 (2013) [V. M. Rozenbaum and I. V. Shapochkina, JETP Lett. 98, 568 (2013)].

9. В.М. Розенбаум, И.В. Шапочкина, Письма в ЖЭТФ 102, 275 (2015) [V.M. Rozenbaum and I. V. Shapochkina, JETP Lett. 102, 248 (2015)].

10. V. M. Rozenbaum, Yu. A. Makhnovskii, I. V. Shapochkina, S.-Y. Sheu, D.-Y. Yang, and S. H. Lin, Phys. Rev. E 92, 062132 (2015).

11. R. D. Astumian and M. Bier, Phys. Rev. Lett. 72, 1766 (1994).

12. F. Jülicher, A. Ajdari, and J. Prost, Rev. Mod. Phys. 69, 1269 (1997).

13. Y. Okada and N. Hirokawa, Science 283, 1152 (1999).

14. В. М. Розенбаум, И. В. Шапочкина, Ё. Тераниши, Л. И. Трахтенберг, Письма в ЖЭТФ 107, 525 (2018).

15. М. А. Кожушнер, Б. В. Лидский, В. С. Посвянский, Л. И. Трахтенберг, Письма в ЖЭТФ 108, 670 (2018).

16. V.M. Rozenbaum, D.-Y. Yang, S.H. Lin, and T. Y. Tsong, J. Phys. Chem. B 108, 15880 (2004). 
17. T. Y. Tsong and R. D. Astumian, Bioelectrochemistry and Bioenergetics 15, 457 (1986).

18. B. Derrida, J. Stat. Phys. 31, 433 (1983).

19. J. C. Skou, Angew. Chem. Int. Ed. Engl. 37, 2321 (1998).

20. T. Y. Tsong and C.-H. Chang, AAPPS Bulletin 13, 12 (2003).

21. G. P. Harmer and D. Abbott, Nature 402, 864 (1999).

22. G. P. Harmer and D. Abbott, Stat. Sci. 14, 206 (1999).

23. J. M. R. Parrondo, G. P. Harmer, and D. Abbott, Phys. Rev. Lett. 85, 5226 (2000).

24. R. Toral, P. Amengual, and S. Mangioni, Physica A 327, 105 (2003).

25. J. M. R. Parrondo and L. Dinis, Contemp. Phys. 45, 147 (2004).
26. В. М. Розенбаум, Химия, физика и технология поверхности 11, 100 (2020).

27. R. D. Astumian, J. Phys. Chem. 100, 19075 (1996).

28. Р. Л. Стратонович, Радиотехника и электроника $\mathbf{3}$, 497 (1958) [Non-linear Transformations of Stochastic Processes, ed. by P.I. Kuznetsov, R. L. Stratonovich, and V. I. Tikhonov, Pergamon Press, Oxford (1965)].

29. R. L. Stratonovich, Theory of Random Noise, Gordon and Breach, London (1969).

30. L. Schimansky-Geier, M. Kschischo, and T. Fricke, Phys. Rev. Lett. 79, 3335 (1997).

31. V.M. Rozenbaum, D.-Y. Yang, S.H. Lin, and T. Y. Tsong, Physica A 363, 211 (2006).

32. V. M. Rozenbaum and I. V. Shapochkina, Phys. Rev. E 84, 051101 (2011). 